\title{
Numerical investigations of the engine cooling system in a small power vessel pod propulsion system
}

\author{
Czesław Dymarski, Prof. \\ Wojciech Leśniewski, Ms. C. \\ Gdansk University of Technology
}

\begin{abstract}
The development of electronics and electrotechnology enabled to put electric motors of the alternating current in the pod and to use them for the main drive of ships. A lot of heat which must be picked up from the system is a problem which is turning up at applying the system of this type. Many big ships lately contended with these problems. Building the small propeller for the boat powered with solar power we decided to check the influence of the chilling middle on the temperature of the work of an engine. In the article we are presenting a built propeller and a way, into which an issue of the exchange of the heat was analysed using the Fluent software.
\end{abstract}

Keywords: azimuthing electric propulsion, pod propeller, heat transfer, cooling system

\section{INTRODUCTION}

Nowadays one of most rapidly developing types of ship propulsion systems is the combined diesel-electric system with two or more azipods. Usually this system consists of a number of current generating units, driven by internal combustion piston engines or gas turbines that deliver power to alternating current motors via the central electric switchgear and frequency converters. The motors, which drive directly ship propellers, are mounted in a pod situated in the lower part of a column suspended under the ship hull. As a rule, the column can move around by $360^{\circ}$ with respect to the vertical axis, which secures excellent manoeuvring abilities of the ship and eliminates the need for using traditional steering gears. The ability to change smoothly the speed of the electric motors makes it possible to use simple and cheap constant pitch propellers. Other advantages of the discussed propulsion system include: smaller space occupied by the system inside the ship, better spatial arrangement of the main engine room elements, higher reliability secured by the use of two or more propellers, and the reduction of noise. These advantages are the reason why more and more ships, in particular large and luxurious passenger ships, and specialised vessels that require dynamic positioning, are equipped with this type of propulsion. It is noteworthy, however, that this type of propulsion, as compared to the traditional system with low-speed internal combustion engines, reveals also certain disadvantages. It is less efficient, much more expensive, and still some technical problems referring to its operation are to be solved. Lower efficiency of the entire system mainly results from lower efficiency of high- or medium-speed internal-combustion engines and the inevitable double energy conversion: from the mechanical energy to the electric energy and vice versa. High investment cost of the system results from extremely high price of motors with magnets made of rare earths, which are to be used here due to their small dimensions that make it possible to install them in the pod. The operating problems mainly refer to the bearing and sealing systems of the electric motor rotor shaft, which simultaneously plays the role of the propeller shaft, and the cooling system used in this motor. It is noteworthy that the above problems referring to the bearing and sealing systems can originate, among other reasons, from incorrect operation of the motor cooling system, as the conditions for heat absorption from the system are extremely unfavourable. Relatively very high power of the motor, against its extremely small dimensions and small space inside the pod, make the process of absorption of the generated heat extremely difficult.

In order to provide opportunities for correct operation of the propulsion system in various operating conditions, numerous research activities are performed as early as at the ship design stage. Due to extremely high cost of experimental investigations, wherever possible they are substituted with numerical analyses that make it possible to simulate system operation in a vast variety of expected operating conditions.

The article presents results of the numerical analysis of operation of the motor cooling system of the low power pod propulsion system, which is to be used in a small vessel, namely the catamaran ENERGA SOLAR that makes use of the solar energy. 


\section{TECHNICAL CONDITIONS AND DESCRIPTION OF THE EXAMINED PROPULSION SYSTEM}

Fig. 1 shows a cross-section through the designed propeller. Its construction differs slightly from that used in high-power propellers. The present construction makes use of a toothed planetary reduction gear, mounted coaxially between the motor and the constant pitch propeller. The kinematic scheme of this gear is given in Fig. 2.

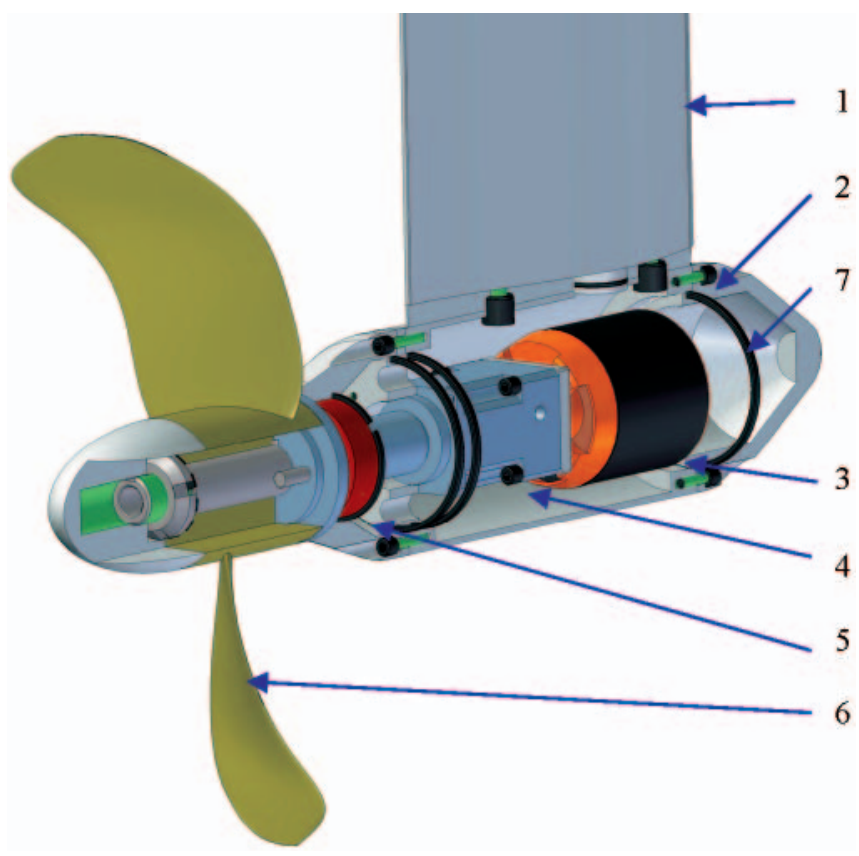

Fig. 1. Cross section through the propeller: 1 -rotating column; 2 -pod; $\mathbf{3}$-electric motor; $\mathbf{4}$ - planetary gear; $\mathbf{5}$-rolling bearing; 6 - constant pitch propeller; 7 -O-ring

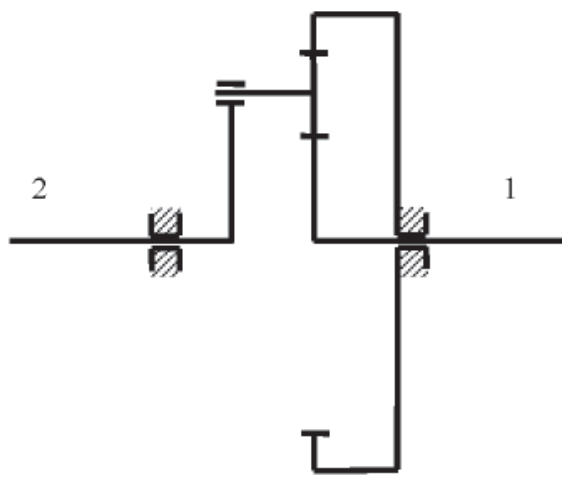

Fig. 2. Kinematic scheme of the planetary gear with transmission ratio $i=3$, made by APEX DYNAMICE, INC. 1 - input shaft; 2- output shaft

The presented propeller uses an asynchronous high torque three-phase motor Axi 5345 made by a Czech company MODELMOTORS Ltd. The nominal torque of the motor is $\mathrm{Mn}=4.5 \mathrm{Nm}$, while its outer diameter is only $63 \mathrm{~mm}$. In order to determine the efficiency of the motor working in combination with the frequency converter, it was examined on a specially prepared laboratory research rig. This examination made it possible to determine efficiency characteristics vs. engine load and rotational speed [1]. For instance, for motor revolutions $\mathrm{n}=30 \mathrm{rev} / \mathrm{s}$ and the torque on shaft $\mathrm{M}=1.1 \mathrm{Nm}$ the system efficiency was equal to $\eta=86.4 \%$ and decreased almost linearly with increased load, down to the level of $\eta=$ $=61.8 \%$ at $\mathrm{M}=4.32 \mathrm{Nm}$.

The applied constant-pitch propeller with $\mathrm{D}=280 \mathrm{~mm}$ in diameter was tested in a natural scale in the flow tank of the Ship Design and Research Centre in Gdansk. These tests made it possible to prepare its hydrodynamic characteristics shown in Fig. 3.

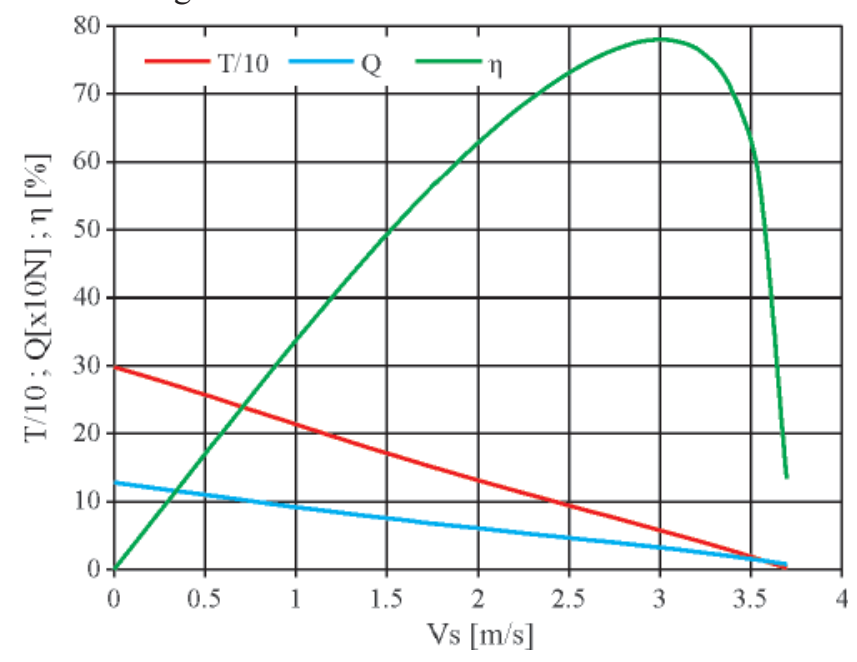

Fig. 3. Hydrodynamic characteristics of the propeller

\section{CALCULATING WATER VELOCITY DISTRIBUTION AROUND THE PROPELLER}

Modelling the flow over the propeller was significant part of the study of the shape of the pod having the electric motor with the gear and bearings installed inside it. It was also part of preparation for heat transfer calculations. The latter task required preparing the data on the velocity field around the pod. More precise methods of this type of calculations are presented, for instance, in [3] and [4]. Moreover, the information about the water flow velocity field in this area makes it possible to estimate, to some extent, the correctness of the assumed pod shape from the hydrodynamic point of view. The presence of strong disturbances in the flow near propeller would indicate that some corrections in the shape of the pod are necessary. To facilitate the activities oriented on preparing the grid that divides the system into finite elements, necessary for numerical calculations, the axial symmetry of the system was assumed which means neglecting the propeller column. This assumption not only made the preparation for numerical calculations much simpler, it also considerably speeded up the calculations themselves. The calculation domain extended $5[\mathrm{~m}]$ both in front of and behind the propeller, and $3[\mathrm{~m}]$ on each side. For the calculated values to model the reality in the most possible way, the calculation grid was refined in the vicinity of the propeller, and was left coarser in the regions in which no significant flow disturbances were expected. The calculations did not take into account the operation of the propeller screw.

The analysis of the flow over the propeller was performed using the code Fluent, a package belonging to a big family of CFD (Computational Fluid Dynamic) codes. This programme makes it possible to calculate velocity and temperature distributions, pressure and mass fields, and model chemical phenomena. It makes use of numerical methods to solve the equations of momentum, energy, and mass transfer. It is based on the finite element method and the finite volume method, the idea of which consists in integrating the equations describing the problem over each control volume. The code provides opportunities for analysing phenomena in two and three dimensions. Given below are the basic equations which are solved by the code when analysing the flow problem. 
Mass conservation equation:

$$
\frac{\partial \rho}{\partial t}+\frac{\partial}{\partial x}\left(\rho v_{x}\right)+\frac{\partial}{\partial r}\left(\rho v_{r}\right)+\frac{\rho v_{r}}{r}=S_{m}
$$

where:

$\mathrm{x}-$ axial coordinate

$\mathrm{r}$ - radial coordinate

$\mathrm{v}_{\mathrm{x}}-$ axial velocity

$\mathrm{v}_{\mathrm{r}}-$ radial velocity

$\mathrm{S}_{\mathrm{m}}-$ added mass

Axial and radial momentum conservation equations:

$$
\begin{gathered}
\frac{\partial}{\partial \mathrm{t}}\left(\rho \mathrm{v}_{\mathrm{x}}\right)+\frac{1}{\mathrm{r}} \frac{\partial}{\partial \mathrm{x}}\left(\mathrm{r} \rho \mathrm{v}_{\mathrm{x}} \mathrm{v}_{\mathrm{r}}\right)+\frac{1}{\mathrm{r}} \frac{\partial}{\partial \mathrm{r}}\left(\mathrm{r} \rho \mathrm{v}_{\mathrm{x}} \mathrm{v}_{\mathrm{r}}\right)= \\
=-\frac{\partial \mathrm{p}}{\partial \mathrm{x}}+\frac{1}{\mathrm{r}} \frac{\partial}{\partial \mathrm{x}}\left[\mathrm{r} \mu\left(2 \frac{\partial \mathrm{v}_{\mathrm{x}}}{\partial \mathrm{x}}-\frac{2}{3}(\nabla \cdot \overrightarrow{\mathrm{v}})\right)\right]+ \\
+\frac{1}{\mathrm{r}} \frac{\partial}{\partial \mathrm{r}}\left[\mathrm{r} \mu\left(\frac{\partial v_{\mathrm{x}}}{\partial \mathrm{r}}+\frac{\partial v_{\mathrm{r}}}{\partial \mathrm{x}}\right)\right]+\mathrm{F}_{\mathrm{r}}
\end{gathered}
$$

and:

$$
\begin{gathered}
\frac{\partial}{\partial t}\left(\rho v_{r}\right)+\frac{1}{r} \frac{\partial}{\partial x}\left(r \rho v_{x} v_{r}\right)+\frac{1}{r} \frac{\partial}{\partial r}\left(r \rho v_{x} v_{r}\right)= \\
=-\frac{\partial p}{\partial x}+\frac{1}{r} \frac{\partial}{\partial x}\left[r \mu\left(\frac{\partial v_{r}}{\partial x}+\frac{\partial v_{x}}{\partial r}\right)\right]+ \\
\frac{1}{r} \frac{\partial}{\partial r}\left[r \mu\left(2 \frac{\partial v_{r}}{\partial r}-\frac{2}{3}(\nabla \cdot \vec{v})\right)\right]+ \\
-2 \mu \frac{v_{r}}{r^{2}}+\frac{2}{3} \frac{\mu}{r}(\nabla \cdot \vec{v})+\rho \frac{v_{z}^{2}}{r}+F_{r}
\end{gathered}
$$

where:

$$
\nabla \cdot \vec{v}=\frac{\partial v_{x}}{\partial x}+\frac{\partial v_{r}}{\partial r}+\frac{v_{r}}{r}
$$

$\mu-$ viscosity

$\mathrm{F}-$ external force

For the processor, which is the package Fluent, to be able to perform the calculations, it needs preparing a relevant computational grid, using a preprocessor, which takes into account boundary conditions. The calculation grid prepared using the preprocessor Gambit for the presently examined problem is shown in Fig. 4.

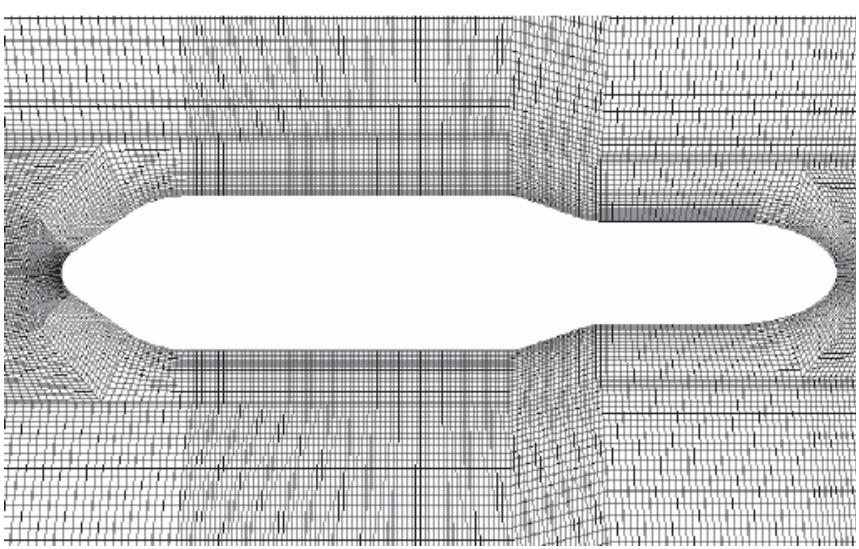

Fig. 4. 2D calculation grid in the vicinity of the propeller
The calculations were performed using as the boundary condition the water velocity equal to $3[\mathrm{~m} / \mathrm{s}]$ at the inlet to the computational domain. The boundaries of this domain were treated as walls without friction generated by the flowing fluid. The distributions of the water velocity magnitude and particular component calculated in the vicinity of the propeller for the above conditions are shown in Fig. 5 .

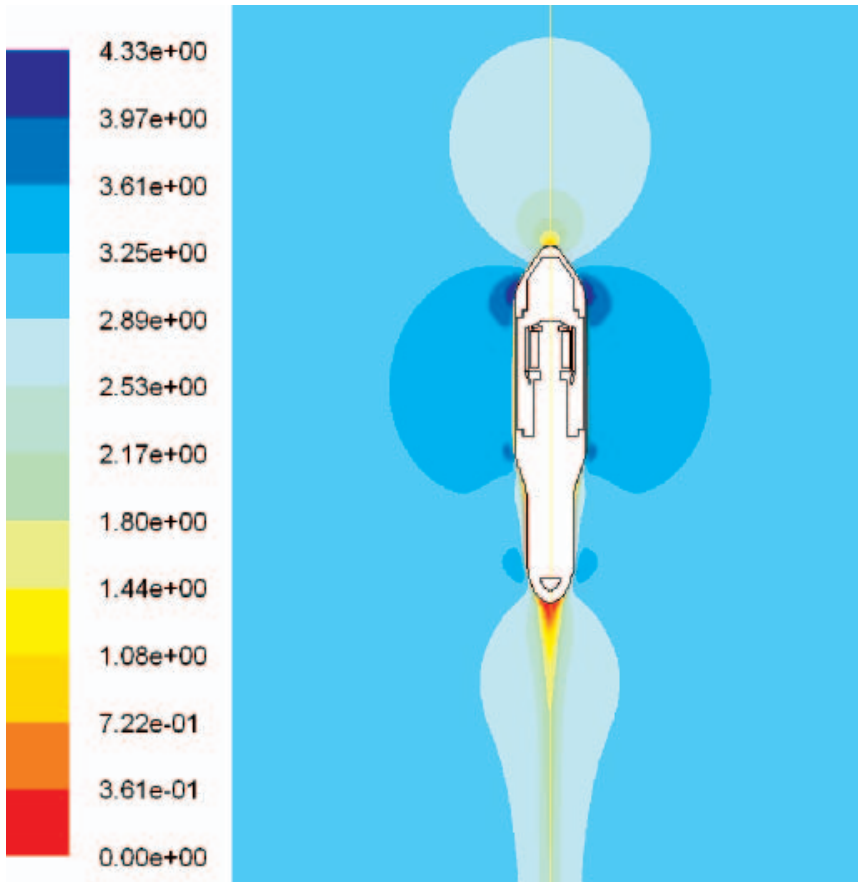

Fig. 5. Velocity distribution in the vicinity of the propeller. Visible is the wake behind the propeller cone

The above-presented results of calculations reveal that the water velocity in direct vicinity of the propeller pod increases from zero at its front end to the maximum level of $4.33[\mathrm{~m} / \mathrm{s}]$ recorded in the border between the conical and cylindrical part. Along the cylindrical part, initially the velocity decreases rapidly and then is stabilised at the level of $3.35[\mathrm{~m} / \mathrm{s}]$, up to the diameter reduction region, where it again increases and decreases below $3[\mathrm{~m} / \mathrm{s}]$.

\section{CALCULATING TEMPERATURE DISTRIBUTIONS IN THE PROPELLER AND SURROUNDING WATER}

A basic problem in the pod propulsion systems concerns absorption of the heat generated by the engine in operation. The heat is generated due to losses that accompany the energy conversion and due to friction processes. Assessing the volume of this heat was possible by measuring, on the research rig, the electric power supplied to the motor and the effective mechanical power on the output shaft for different loads. For instance, for the propeller filled with oil and the mechanical power output $\mathrm{P}_{\mathrm{e}}=500$ [W] the power loss, equivalent to the flux of generated heat, was approximately equal to $\mathrm{P}_{\text {str }}=150[\mathrm{~W}]$.

Finite element method based heat transfer calculations were in this case much more difficult due to the type of the examined motor with rotating case, which required preparation of a $3 \mathrm{D}$ calculation grid. In order to decrease the labour consumption and shorten the time of calculations the modelled shape was limited to one fourth of the propeller. It was assumed additionally that the heat generated in the motor is carried away to the water flowing round the propeller only through the cylindrical surface of the pod. 
Heat transfer calculations, performed with the aid of the FEM method, were much more complicated in this case due to the type of the examined motor, which was the motor with rotating casing. The basic energy equations solved by the code are given below.

$$
\begin{gathered}
\frac{\partial}{\partial \mathrm{t}}(\rho \mathrm{E})+\nabla \cdot(\overrightarrow{\mathrm{v}}(\rho \mathrm{E}+\mathrm{p}))= \\
=\nabla \cdot\left(\mathrm{k}_{\mathrm{eff}} \nabla \mathrm{T}-\sum_{\mathrm{j}} \mathrm{h}_{\mathrm{j}} \overrightarrow{\mathrm{J}}_{\mathrm{j}}+\left(\overline{\bar{\tau}}_{\mathrm{eff}} \cdot \overrightarrow{\mathrm{v}}\right)\right)+\mathrm{S}_{\mathrm{h}}
\end{gathered}
$$

Where: the three first terms on the right-hand side of the equation represent, respectively, the energy transfer due to conduction, diffusion and dissipation. $\mathrm{S}_{\mathrm{h}}$ represents chemical reaction heat and any other source of heat.

$$
E=h-\frac{p}{\rho}+\frac{v^{2}}{2}
$$

For a solid body:

$$
\begin{aligned}
& \quad \frac{\partial}{\partial \mathrm{t}}(\rho \mathrm{h})+\nabla \cdot(\vec{v} \rho \mathrm{h})=\nabla \cdot(\overrightarrow{\mathrm{u}} \rho \mathrm{h})=\nabla \cdot(\mathrm{k} \nabla \mathrm{T})+\mathrm{S}_{\mathrm{h}} \\
& \rho-\text { density } \\
& \mathrm{h}-\text { enthalpy } \\
& \mathrm{k}-\text { conductivity } \\
& \mathrm{T}-\text { temperature }
\end{aligned}
$$

The calculations required preparing a 3D grid. In order to optimise the labour and time consumption of the calculations, the modelled shape was limited to one fourth of the propeller. Moreover, it was assumed that the heat generated in the motor is transferred to the water flowing by the propeller only through the cylindrical part of the pod.

The calculation grids for the examined flow region and propeller elements are given in Figs. $6 \div 9$.

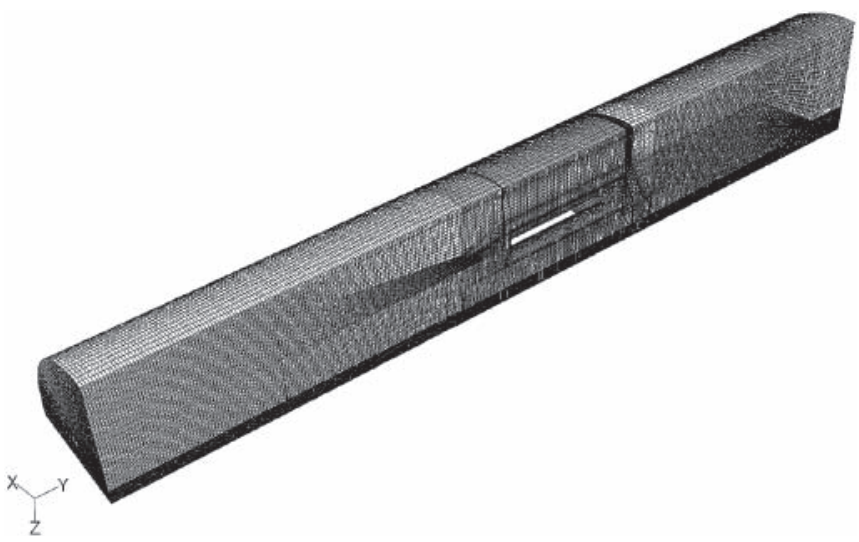

Fig. 6. $3 D$ calculation grid for the examined heat transfer region (without water flowing by the propeller)

It was assumed that the entire heat is generated in electric motor windings. In the prepared calculating model it was assumed that the heat flux originates on inner walls of the winding, having the surface $\mathrm{S}=0.006\left[\mathrm{~m}^{2}\right]$. For the heat flux generated at effective power output $\mathrm{P}_{\mathrm{e}}=500[\mathrm{~W}]$ and equivalent to the power loss $\dot{\mathrm{Q}}=\mathrm{P}_{\text {str }}=150[\mathrm{~W}]$ the heat flux density $\dot{\mathrm{q}}$ attributed to the examined quarter of the calculation domain is equal to:

$$
\dot{\mathrm{q}}=\frac{\dot{\mathrm{Q}}}{4 \cdot \mathrm{S}}=\frac{150}{4 \cdot 0.006}=6250\left[\mathrm{~W} / \mathrm{m}^{2}\right]
$$

The input parameters for the calculation were:

$\mathrm{V}$ - velocity of the water flowing by the propeller, calculated in the way presented in Chapter 2 n - rotational speed of the motor

$\dot{\mathrm{Q}}$ - heat flux generated by the motor, determined from the efficiency measured on the research rig

$T_{w}$ - temperature of the water flowing by the propeller.

along with the following properties of the oil used for motor cooling:

$\rho \quad$ - oil density

Cp - oil specific heat

$v$ - oil kinematic viscosity coefficient

$\alpha$ - overall heat-transfer coefficient.

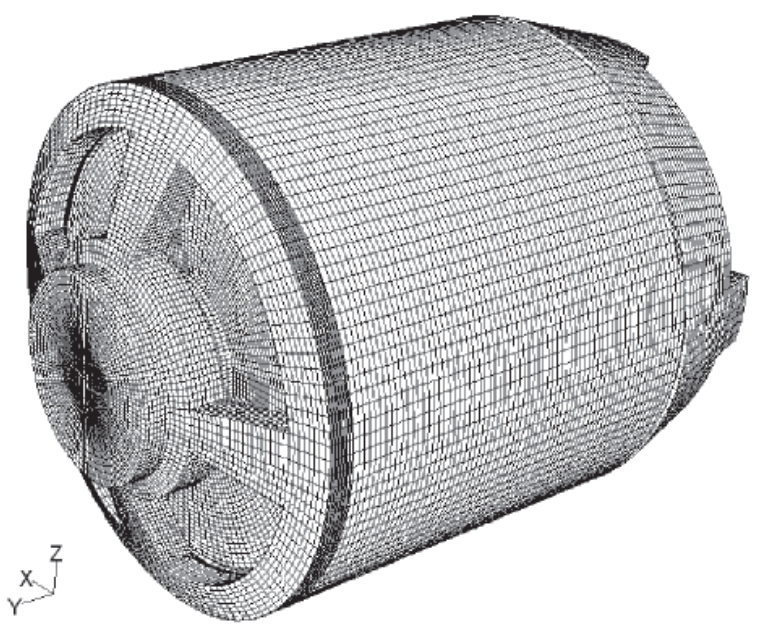

Fig. 7. 3D calculation grid modelling the motor

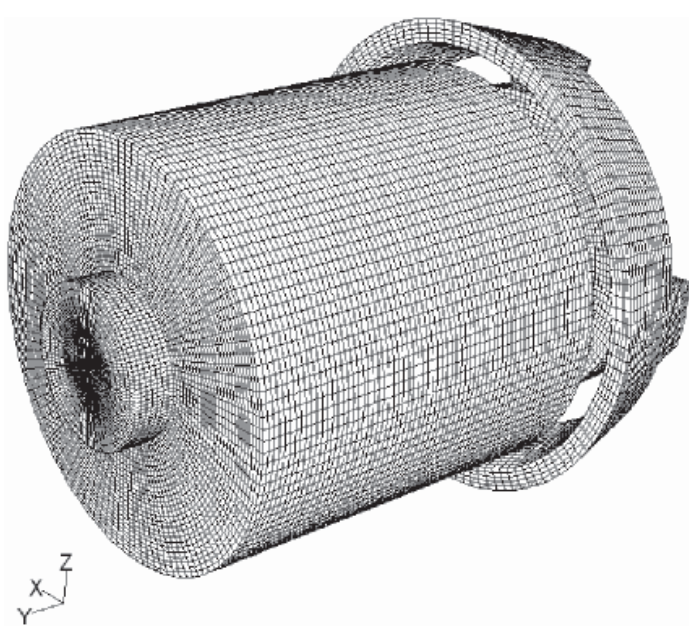

Fig. 8. 3D calculation grid modelling the motor stator

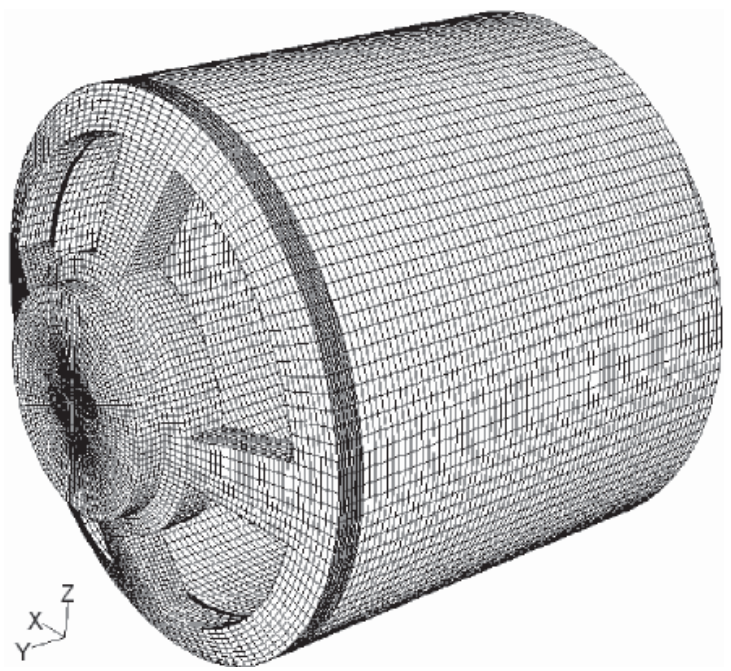

Fig. 9. 3D calculation grid modelling the motor rotor 
Current values of the above parameters which were used in the calculations were the following:

$\begin{aligned} \mathrm{V} & =3.35 & & {[\mathrm{~m} / \mathrm{s}] } \\ \mathrm{n} & =30 & & {[\mathrm{rev} / \mathrm{s}] } \\ \dot{\mathrm{Q}} & =150 & & {[\mathrm{~W}] } \\ \mathrm{T}_{\mathrm{w}} & =291 & & {[\mathrm{~K}]\left(18\left[{ }^{\circ} \mathrm{C}\right]\right) } \\ \rho & =857 & & {\left[\mathrm{~kg} / \mathrm{m}^{3}\right] } \\ \mathrm{Cp} & =2160 & & {[\mathrm{~J} / \mathrm{kgK}] } \\ \nu & =0.026 & & {[\mathrm{~kg} / \mathrm{ms}] } \\ \alpha & =0.2 & & {[\mathrm{~W} / \mathrm{mK}] }\end{aligned}$

Fig. 10 presents the calculated distributions of linear velocities in selected propeller elements.

Temperature distributions calculated for the above input data in the examined region and in the cylindrical part of the pod are shown in Figs. 11 and 12.

The above presented distributions reveal that the maximum temperature takes place in the motor iron region and is equal to $\mathrm{T}_{\max }=301[\mathrm{~K}]\left(28\left[{ }^{\circ} \mathrm{C}\right]\right)$. The heat from the iron is taken by the gear, rotor and oil. To assess the contribution of the cooling medium which fills the propeller motor and pod, identical calculations were performed for the same conditions when the

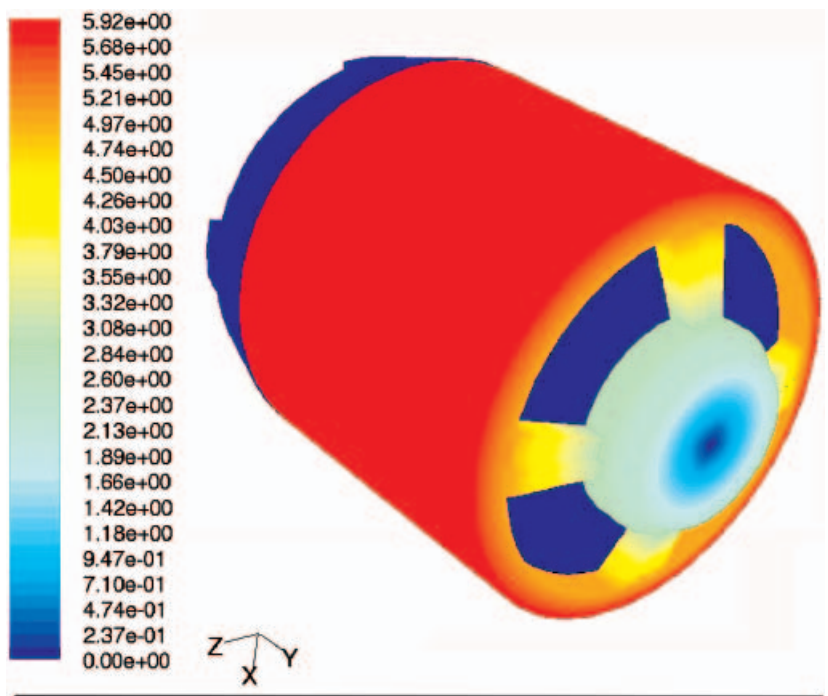

Contours of Velocity Magnitude ( $\mathrm{m} / \mathrm{s}$ )

Sep 05, 2007 FLUENT 6.2 (3d, segregated, ske)

Fig. 10. Linear velocity distribution in motor elements

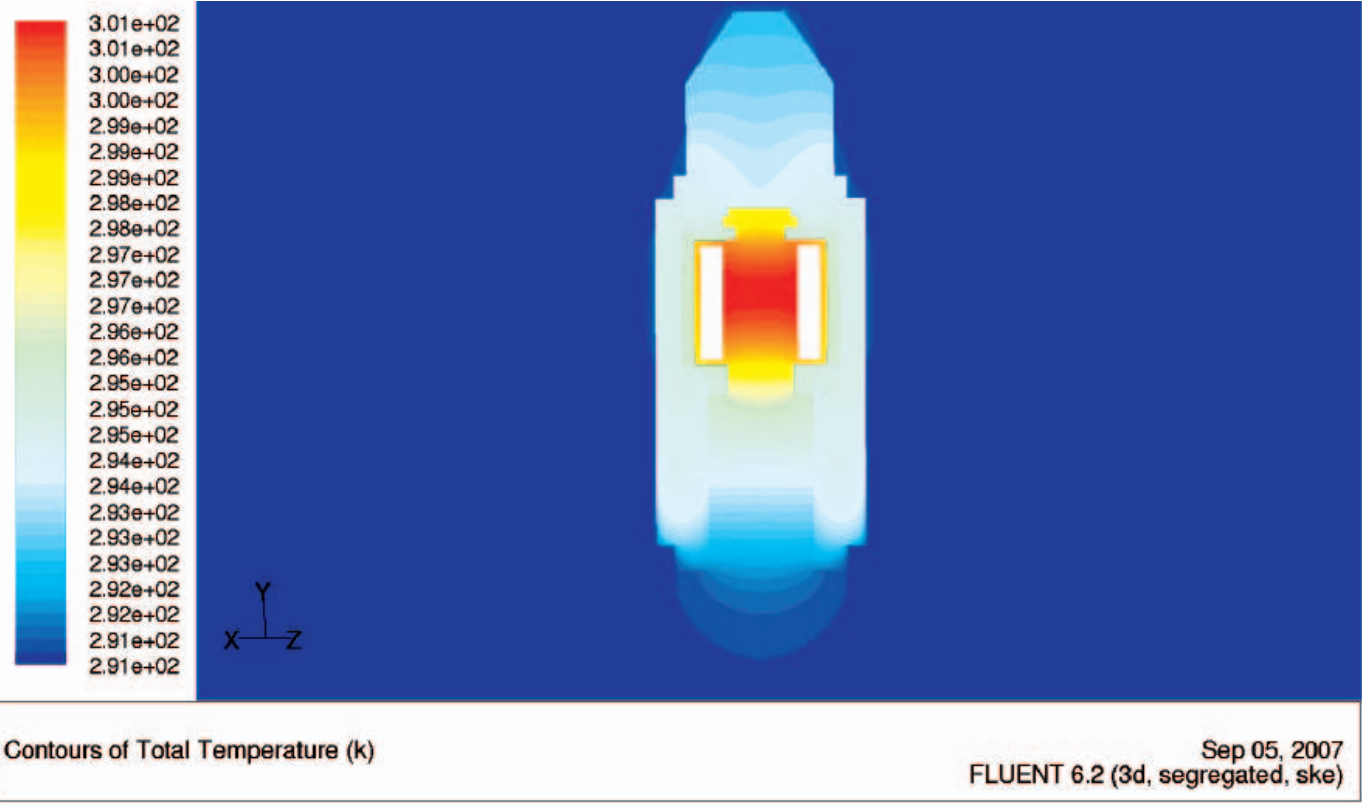

Fig. 11. Temperature distribution inside the propeller filled with oil
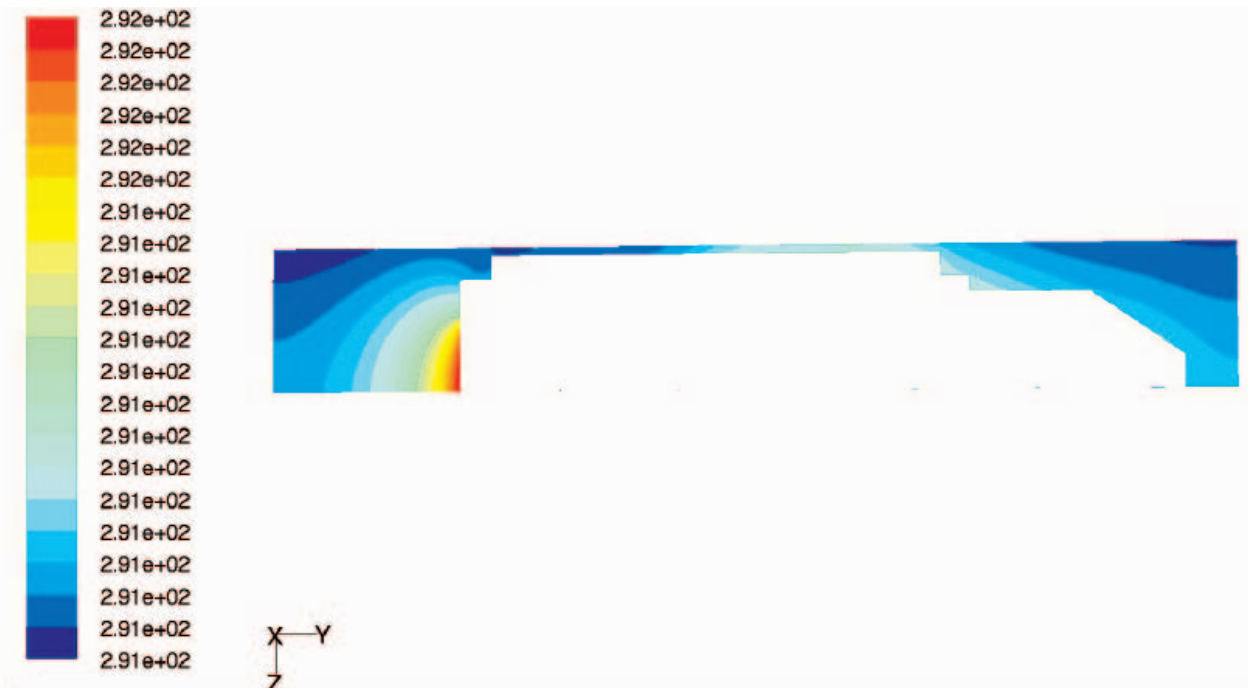

$2.91 \theta+02$

$2.910+02$

$2.910+02$

910+02

$2.91 \theta+02$

Fig. 12. Temperature distribution on walls composing the cylindrical part of the propeller pod 


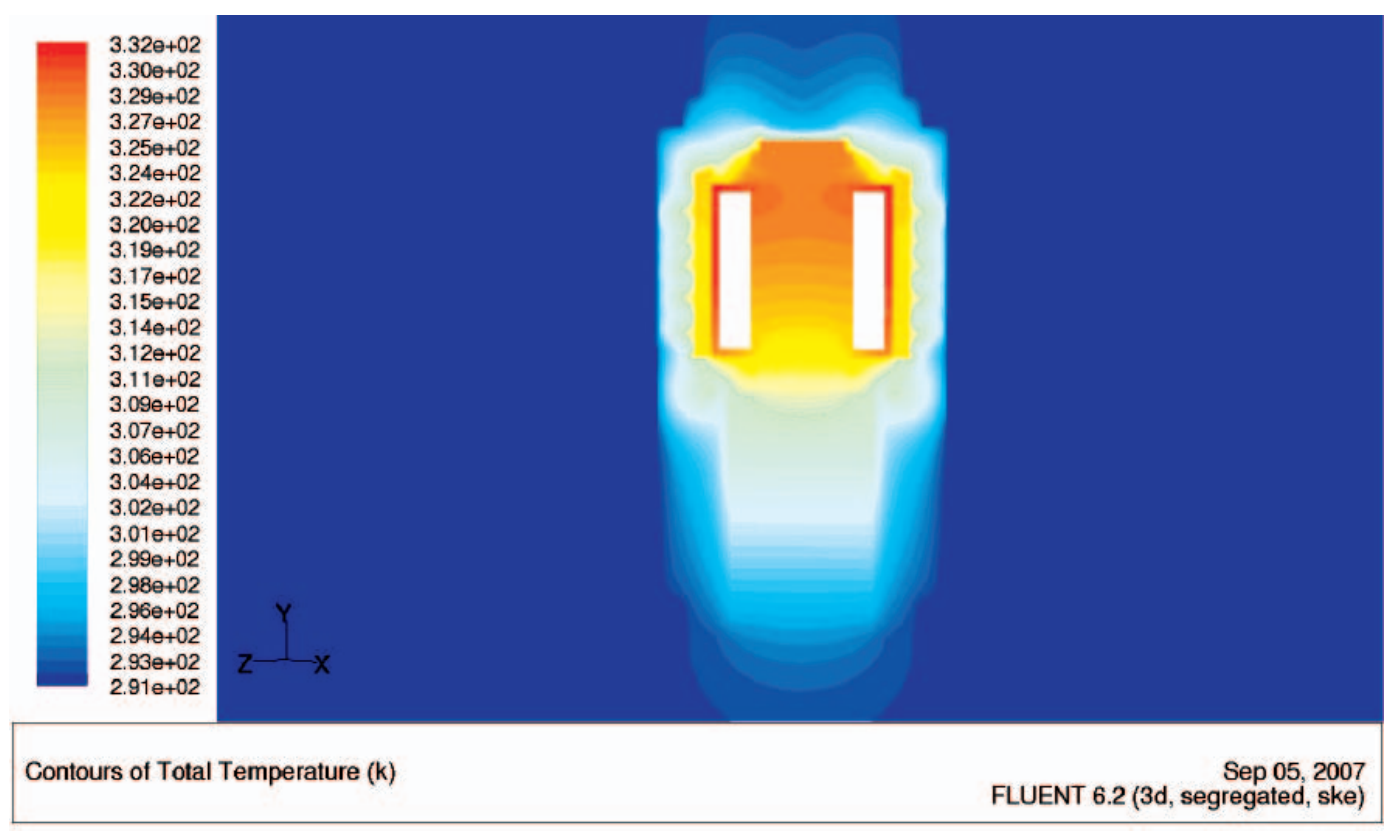

Fig. 13. Temperature distribution inside the propelled filled with the air

propeller was filled with the air instead of oil. The results of those calculations are shown in Fig. 13. As could have been expected, the air was not capable of taking over as much heat as the oil. The maximum temperature in this case reached $\mathrm{T}_{\max }=332[\mathrm{~K}]\left(59\left[{ }^{\circ} \mathrm{C}\right]\right)$.

\section{CONCLUSIONS}

The designs of electric propulsion systems for small boats which are available on the market are the attached direct current motors that reveal small overall efficiency. They are used in general as auxiliary propulsion system and could not be used in the constructed racing catamaran driven by solar energy as they cannot work continuously for such a long time. The sailing races for which the boat Energa Solar was designed provide opportunities for presenting own technical solutions and comparing them with those presented by leading teams from all over the world. Energa Solar is the only boat to have the propulsion system based on such a small three-phase motor, which makes it possible to enter upon competition with strong teams from all over the world.

The performed investigations of the propeller screw and motor allowed a relevant planetary gear to be selected. The determined characteristics have proved that the motor can operate in a direct drive system. However, power losses recorded in such a design are many times as high as those generated by the planetary gear. In this case the motor operating at the rotational speed $11[\mathrm{rev} / \mathrm{s}]$ should be loaded with a torque three times as high as that recorded in the proposed design. The losses recorded in the motor working in combination with an inverter would reach as much as $70 \%$. The presently produced planetary gears are precise machines, the efficiency of which reaches $97 \%$ even at very small power levels.

O Manoeuvring such a small vessel on the water forces frequent changes of operating parameters of the propulsion system. Instantaneous losses can be higher than those assessed during the investigations. Consequently, the oil temperature in the propeller can also rise higher. The use of oil as a cooling medium results from experience gained during the racings.
The temperature determined in the numerical analysis is verisimilar taking into account the results of temperature measurements performed on the motor working in oil.

The next step in the research will be the measurement of temperature distributions on a real object. The designed propeller, shown in Fig. 14, will also be examined in respect of the effect of the propeller screw position on the overall efficiency of the propulsion system.

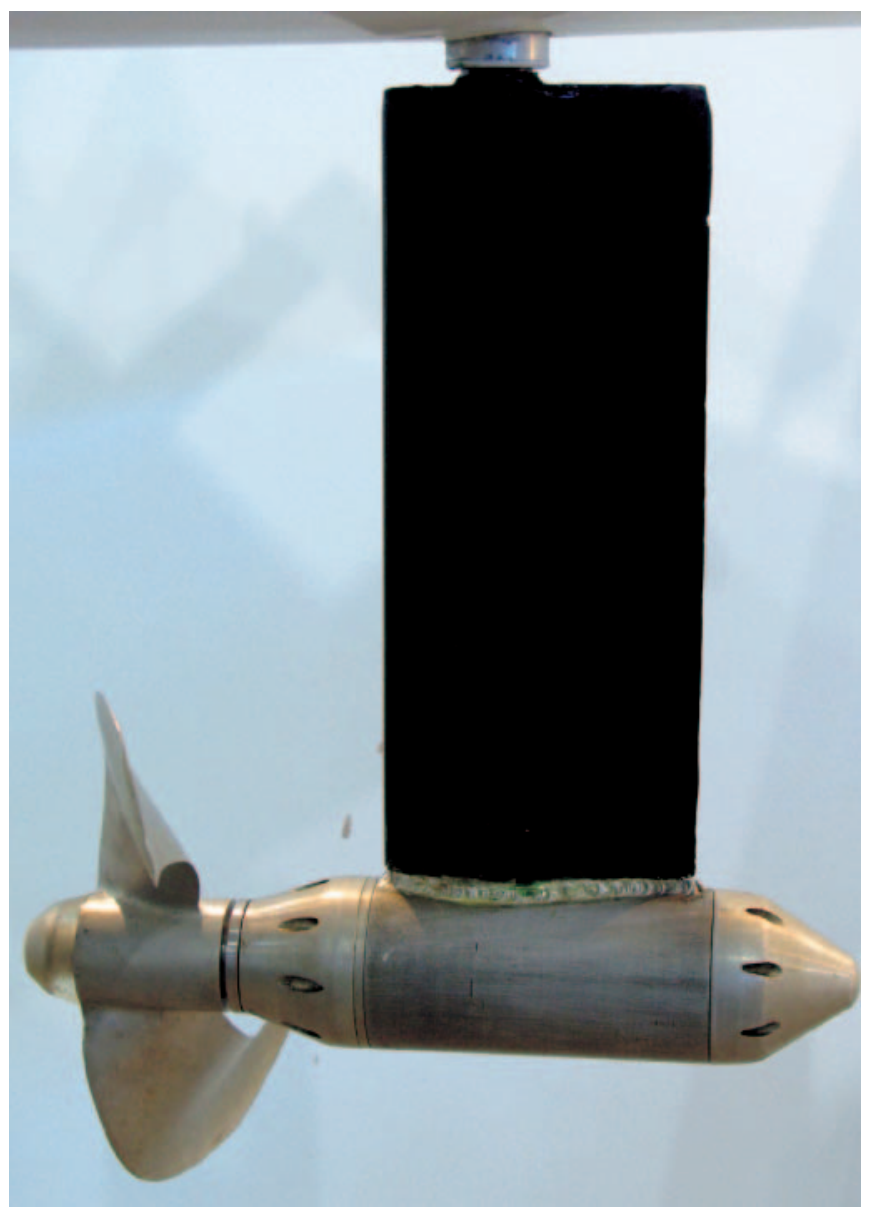

Fig. 14. The designed propeller in the arrangement with the pulling screw 
Due to permanent increase of oil prices, the electric drive becomes an alternative for internal combustion engines. Studying problems concerning ecological propulsion systems is very popular and frequent nowadays. More and more companies assign large sums for developing initiatives promoting the use of renewable energy resources.

\section{BIBLIOGRAPHY}

1. Chachulski Kazimierz: Fundamentals of ship propulsion (in Polish), Wydawnictwo Morskie, Gdańsk 1988

2. Dudziak J.: Theory of ship (in Polish), Foundation for the Promotion of Maritime Industry. Second issue, updated, Gdańsk 2008

3. Dymarski P., Dymarski Cz.: Curvilinear Panels and Higher Order Dipole Distribution Method for Ducted Propeller Flow Calculations. Marine Technology Transaction, Technika Morska, Polish Academy of Sciences - Branch in Gdańsk, Marine Technology Committee. Vol. 12, $2001 \mathrm{r}$.

4. Dymarski P.: Numerical simulation of viscid flow around hydrofoil, Polish Maritime Research, No 1/2006

5. Collective work edited by M. Dietrich: Fundamentals of machine construction. Third edition, Wydawnictwa Naukowo Techniczne Warszawa 2007
6. Niezgodziński M. E., Niezgodziński T.: Resistance formulas, diagrams and tables, Wydawnictwa Naukowo Techniczne Warszawa 1996 (in Polish)

7. SKF catalogues of products

8. APEX DYNAMICS, INC catalogues of products

9. MODELMOTORS LTD catalogues of products

10.SHOTTEL catalogues of products

11. WARTSILA catalogues of products

12.ROLLS-ROYCE catalogues of products

13.FLUENT 6.0 Documentation.

\section{CONTACT WITH THE AUTHORS}

Czesław Dymarski, Prof.

Wojciech Leśniewski, Ms. C.

Faculty of Ocean Engineering and Ship Technology,

Gdańsk University of Technology Narutowicza 11/12

80-952 Gdańsk, POLAND

e-mail : cpdymars@pg.gda.pl

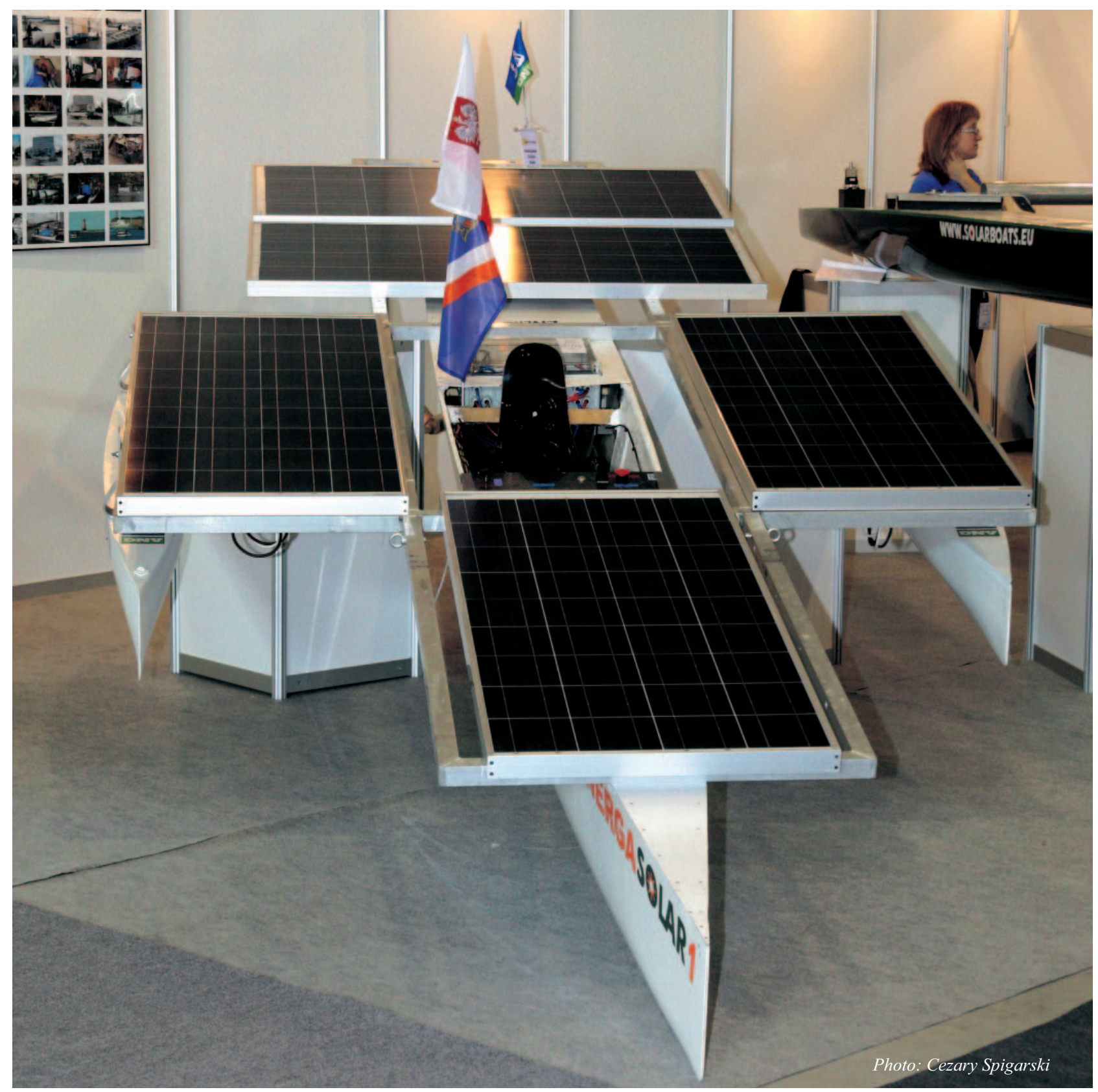

